\title{
Impact on the Regional Ambulance Services and Emergency Departments of the 2017 Freezing Rain in Milan
}

\author{
Giovanni Landoni ${ }^{1, *}$, Omar Saleh ${ }^{1}$, Maurizio Migliori ${ }^{2}$, \\ Carlo Picco ${ }^{2}$
}

\author{
${ }^{1}$ Department of Anaesthesia and \\ Intensive Care, IRCCS San Raffaele \\ Scientific Institute, via Olgettina 60, \\ 20132 Milan, Italy \\ ${ }^{2}$ Azienda Regionale Emergenza \\ Urgenza, Via Alfredo Campanini 6, 20124 \\ Milan, Italy \\ *Correspondence \\ landoni.giovanni@hsr.it \\ (Giovanni Landoni)
}

\begin{abstract}
Background: Freezing rain, also known as gelicidium, glaze, or ice storm, is an unpredictable and rare meteorological event with an impact on millions of citizens and which can affect the healthcare system of large areas. It occurs if rain hits the ground when surface temperatures are below $0{ }^{\circ} \mathrm{C}$. Objective: To report what occurred on January 13th 2017 in Lombardy, a northern Italy region with Milan as capital, when a freezing rain caused an overload of the emergency system. Methods: We obtained data on emergency vehicles calls and missions from the emergency reports (four Lombardy areas covered by Azienda Regionale Emergenza e Urgenza-AREU-and encompassing 117 emergency departments). Emergency examinations data included overall visits, diagnosis, severity degrees and workload of emergency departments. Results: The Milan area emergency calls had an increase unseen in the previous four years of service (from 1369 to $2700 \mathrm{p}<0.001)$. A total of 1913 trauma cases were managed versus a daily average of 484 during January 2017 ( $p<0.001$ ). Road accidents and injuries at work increased while non-traumatic causes did not. More than $70 \%$ of the 38 emergency departments in the Metropolitan Area around the city of Milan were overloaded. Conclusion: We presented the first European report on the impact of an ice storm on a healthcare system, suggesting that early recognition, communication between meteorologists and health authorities, and an alert of ambulance services and healthcare professionals (orthopaedics above all) is mandatory, jointly with warnings to the population by public authorities.
\end{abstract}

\section{Keywords}

Freezing rain, Glaze, Ice storm, Emergency medical service, Ambulance service, Emergency department

\section{Introduction}

Freezing rain, also called glaze or ice storm, is an uncommon weather phenomenon characterized by rain falling when surface temperature is below freezing point $\left(0^{\circ} \mathrm{C}\right.$ or $32{ }^{\circ} \mathrm{F}$ ) [1]. During winter in temperate regions, freezing rain occurs when an originally frozen precipitation meets a warm air front. If rain hits the ground when surface temperatures are below $0{ }^{\circ} \mathrm{C}$, it may instantly freeze, coating anything it touches in a thick film of ice [2]. This very slick layer can pose a serious threat to pedestrians and vehicles in large areas, thus potentially causing a critical medical scenario. Evidence is almost exclusively observational and scattered across decades due to the relative rarity of the event.

Freezing rain can pose a serious threat if it hits a large urbanized area. One of such events happened on January 13th 2017 in the Region of Lombardy, in northern Italy (Lombardy) around the city of Milan, leading to a massive mobilization of the regional emergency medical service (Azienda Regionale Emergenza Urgenza, AREU) and causing severe difficulties to emergency departments 


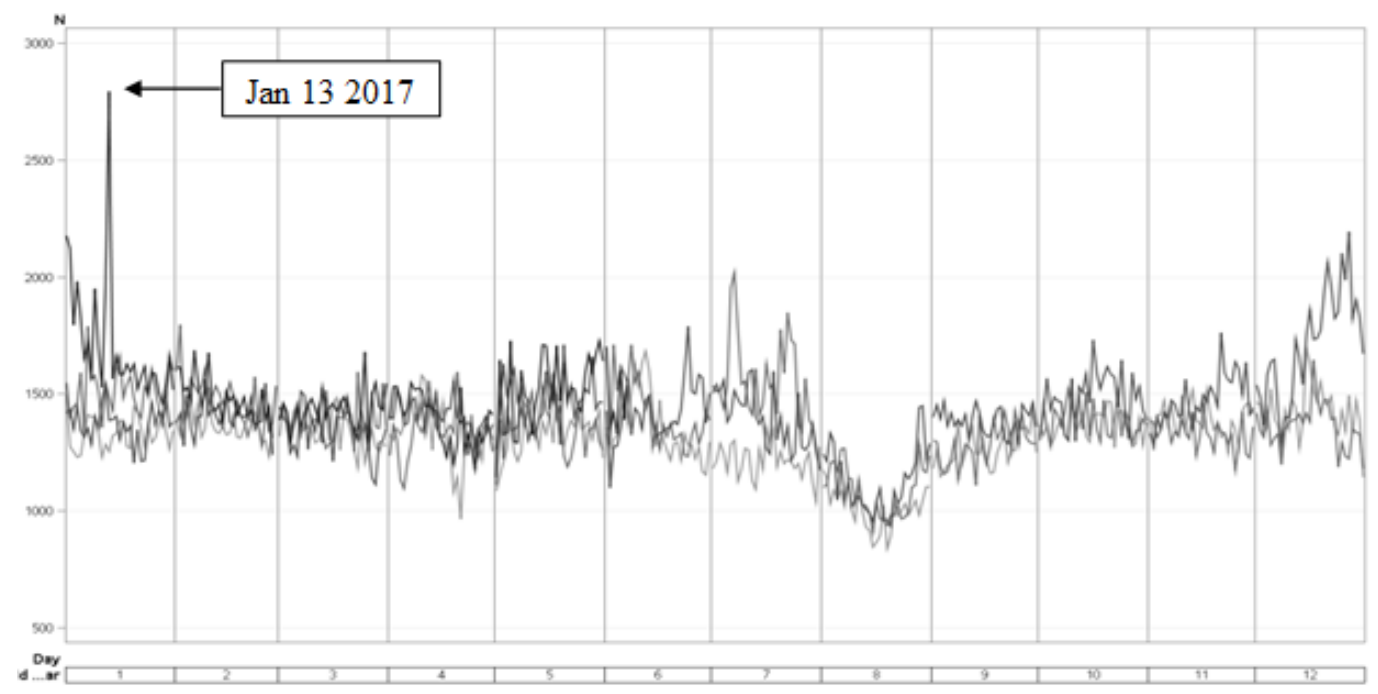

F I G U R E 1. Emergency Calls in the Metropolitan Area of Milan (38 EDs, covering a population of 4,099,521) between January 1st 2014 and May 31st 2017, showing a peak on January 13th 2017. The drop in the number of emergency calls during the month of August is due to the habitual summer holiday period in Italy.

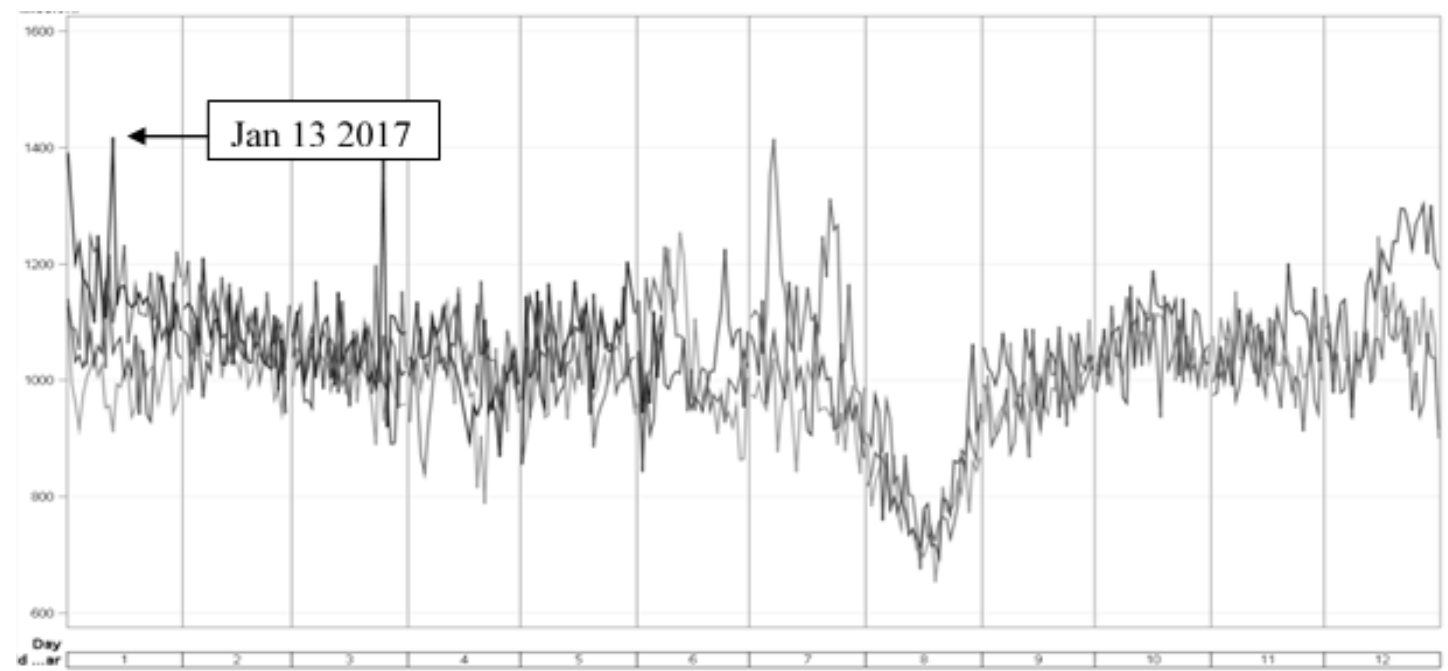

F I G U R E 2. Emergency Missions in the Metropolitan Area (38 EDs, covering a population of 4,099,521) between January 1st 2014 and May 31st 2017, showing a peak on January 13th 2017 (freezing rain), March 2017 (the Pope visit to Milan) and July 2015 (heat wave). The drop in emergency missions during the months of August is due to the habitual summer holiday period in Italy.

(ED) in the major hospitals in the area. Extensive use of the emergency network in a major urban centre increases the need for medical personnel and hospital beds, with unpredictable effects on the medical system as a whole.

We report the effect of a freezing rain event on the number and quality of emergency calls in the city of Milan, the largest urban area in northern Italy, and surrounding area (i.e. Metropolitan Area of Milan, MM). Data about admissions in all EDs and the overload of interested EDs in Lombardy are also reported to measure the effective workload imposed on the medical system.

\section{Materials and Methods}

Meteorological data about the area under study were obtained from official records by the regional institute of environmental policies in Northern Italy (ARPA - Agenzia Regionale per la Protezione Ambientale) and from meteorological bulletins published on newspapers and the Internet. Data on all emergency calls to the 1.1.2. European Emergency Number and rescue missions in Lombardy were retrieved from official AREU activity reports. Daily ED admissions were retrieved from the regional E.U.O.L. system (Emergency and Urgency On Line).

The Ethical committee approval was waived according to the Italian law for observational data already anonymised at data collection. 
AREU manages a fleet of over 250 ambulances with volunteers and employees belonging to rescue Agencies, Associations and Social Cooperatives, 100 Advanced Life Support (ALS) vehicles with doctors or nurses on board, and 5 helicopters covering the whole of Lombardy 24 hours a day. AREU is part of the out of hospital emergency service $(\mathrm{OOH})$ which guarantees national assistance providing adequate interventions in all health emergency events, either to individual persons or in multiple emergencies. The assistance is ensured 24 hours a day throughout the year. The Lombardy AREU was born on April 2nd 2008 (Regional Government Council Resolution VIII/6994) to promote the $\mathrm{OOH}$ emergency service progress-through the integration of $\mathrm{OOH}$ and in-house ED services.

The study period for emergency calls and subsequent dispatch of emergency teams was defined as from January 1st 2014 to May 31st 2017. In order to compare trends over time between different years, all calls and missions were reported in a timeline chart, each line referring to a single year.

For emergency department admissions we reduced the study period from January 1 st to January 31 st 2017 . The following data were retrieved for each one of the 117 EDs: total admissions, colour coded severity of the admission (i.e. white, green, yellow, and red corresponding to increasing severity), admission diagnosis at triage and location of the traumatic event, where applicable. Data were reported in a timeline chart to show monthly trends, or in a table comparing data relative to the day of the event with the monthly average.

Finally, we introduced a "burden index" which enabled to measure the workload imposed on each ED, limitedly to admissions of patients who were held for further management in either the ED or the hospital. Based on a normal distribution of cases derived from historical data for each ED, a particular ED was defined as overloaded if the total of admissions on a given day exceeded the 91th centile of the said distribution. The average workload of each ED was also measured by means of a time chart reporting the average waiting time in the single EDs, as well as the total time spent there by each patient.

Student's t-test was used to compare the weighted mean of calls and missions during January 2017 to the mean calls and missions during the same month in the three preceding years. Shapiro-Wilk's skewness test was used to compare all ED admissions and trauma cases managed in the EDs on the event day versus the average daily cases during the same month. All data were stored electronically and analysed using Microsoft Excel (Microsoft Office Professional Plus v.15.0.4420.1017, 2013) and STATA 2013 (StataCorp LP v13.0 for Windows 64-bit x86-64).

\section{Results}

We found from meteorological bulletins that the MM area was the most affected by the freezing rain event on January 13th 2017 in comparison to the other 3 areas covered by the AREU service in Lombardy (supplemental Fig. 1). The
MM area includes a total of 38 EDs, covering a population of 4,099,521 while the entire Lombardy area includes 117 EDs, with a population of 10,028,904.

AREU professionals involved in the crisis at the time report that the severity of the situation peaked around 10 am and slowly waned by sundown (occurring around $5 \mathrm{pm}$ in January).

During the 4 year-study period, the service received an average of 1369 calls a day in the MM area. On the day of the event, the number of emergency calls in this area (Fig. 1) increased to over $2700(\mathrm{p}<0.001)$, and a lower increase of calls over the daily average was registered in the other areas served by AREU in Lombardy (supplemental Fig. 2).

Over the 4 years under study, an average of 1023 missions per day were dispatched in the MM area following to emergency calls. By contrast, on January 13th 2017, more than 1400 missions (Fig. 2) were dispatched in the same area $(\mathrm{p}<0.001)$. Notably, the other peaks in the chart were registered on March 2017, in coincidence with the Pope's visit to Milan, and on July 2015, when an exceptional heat wave hit the city. Very low or no peaks were registered elsewhere in Lombardy (Supplemental Fig. 3)

Concerning EDs, 6443 admissions were managed in the MM area on January 13th 2017, versus a daily average of 4434 cases during January 2017 ( $p<0.001$ ), with a 45\% increase (supplemental Fig. 4). The severity of ED cases during the day of the event was mostly moderate to low (supplemental Fig. 5). Trauma was the leading cause of the rise in ED admissions, particularly in the Metropolitan area (supplemental Fig. 6). A total of 1913 trauma cases were managed on the event day, versus a daily average of 484 cases during the other days of January 2017 ( $p<0.001$ ). The increase was imputed to road accidents and injuries at work (supplemental Fig. 7). The total number of ED admission due to non-traumatic causes was not higher than the monthly average (supplemental Table 1).

The ED overload during the period of interest is shown in (supplemental Fig. 8) of the supplementary material. Notably almost $70 \%$ of the EDs located in the city of Milan were overloaded. Time charts reporting the average waiting time in the single EDs, as well as the total time spent there by each patient, are reported in (supplemental Fig. 9) of the supplementary material.

\section{Discussion}

We report an extraordinary increase in emergency calls, missions, ED accesses and overload in the Milan area during an ice storm. The data suggest that a swift alert of the ambulance service and healthcare professionals (orthopaedics above all) is mandatory when this situation occurs, jointly with warnings to the population by public authorities.

Albeit uncommon, freezing rain is fearsome as it can hit hard, fast and almost unpredictably. The events here described occurred on January 13th 2017, and was outstanding for its extent and severity in a short timeframe. Similar events of comparable magnitude hit the region only 
in 2009 and 1999.

The AREU service was conferred the Outstanding Emergency Call Centre award by the European Emergency Number Association in 2015 for its unparalleled connection and capability of rapid response [3] and is recognized as one of the best ambulance services in Europe. On January 13th 2017 the situation was so critical in the city that calls exceeded the ability of the ambulance service, forcing the AREU service to resort to private vehicles in some areas. This has important implications in the perspective of a future recurrence. The event may be unpredictable, but a stronger communication between meteorologists and the AREU headquarters may be useful to centralize ambulances where the event is hitting harder.

Most of the emergency departments in the city were overloaded. Notably, since most cases were green- and yellow-coded traumas, i.e. of low severity, alerting a consistent number of trauma management expert personnel may be a winning strategy to lower ED workload in future events.

This meteorological phenomenon can happen worldwide but recent publications are scarce. The only reports on freezing rain events published in the last 20 years are from North America, including the winter storm report of Illinois (1998) [4], the impact of the 1996 blizzard in New York [5], the North Carolina ice storm in 2002 [6], the study of the 2007 Oklahoma winter storm [7] and the 2013 ice storm in Ontario [8]. All these studies considered an ice storm that caused freezing rain and lasted for several days.

The Illinois, Oklahoma and Ontario studies consistently found an increase in injuries from falls. Injuries from pedestrian falls were the most observed, with injuries due to vehicle crashes being the second most commonly reported. The Oklahoma study also reported that injuries were mostly of low severity. The Oklahoma and Ontario studies best match the region-wide, population-based design used in our study and their findings are consistent with ours. Moreover, these studies found certain types of injuries to be more common during the storm aftermath: vehicle crashes peaked in the first days, while falls and injuries related to cleaning saw a delayed increase. This is not applicable to our study because the event we consider lasted one day and did not provoke significant environmental damage.

On the other hand, the New York blizzard study and the North Carolina ice storm study differ in their reporting of an increase in non-traumatic events during an ice storm, mainly provoked by cold, falling debris and power outages which caused several cases of carbon monoxide poisoning due to misuse of heating devices. This is again not applicable to our analysis because freezing rain hit Milan over a single day.

The strength of our study comes from the vast, population-based amount of data from the very efficient emergency service active in Lombardy. Moreover, we had access to data from the ambulance service and from 117 EDs (38 in the city of Milan alone), making this study the most extensive and most comprehensive report on the entire emergency procedure applied during a freezing rain event. The relationship between emergency calls, missions and ED admissions may not be immediate as people often refer to the ED by their own means rather than by ambulance. Furthermore, our estimate of ED overload is not a validated index.

We presented the first European report on the impact of an ice storm on a healthcare system, suggesting that early recognition and alert are the initiatives to be taken for such a rare event. This event must be viewed as an emergency, not because of the severity of the damage it provokes, which is relatively low, but rather because it puts the system under such pressure that any other "true" concurrent emergency may have catastrophic consequences.

\section{ACKNOWLEDGMENTS}

The authors acknowledge the institutional support from AREU for sharing its data and the help of many people who helped editing this work.

\section{CONFLICT OF INTEREST}

There is no conflict of interest.

\section{FUNDING SOURCES}

No public or private funding source was involved in the study.

\section{REFERENCES}

[1] American Meteorological Society Glossary - Glaze [Internet]. [cited 2017 May 7]. Available from: http://glossary.ametsoc.org/ wiki/Glaze.

[2] Freezing Rain: supercooled droplets freezing on impact [Internet]. [cited 2017 May 7]. Available from: http://ww2010.atmos, uiuc.edu/(Gh)/guides/mtr/cld/prcp/zr/frz.rxml.

[3] EENA - 112 Awards 2015 - The stories of the awardees [Internet]. [cited 2017 May 16]. Available from: http: //www . eena.org/pages/112-awards-2015---thestories-of-the-awardees\#. WRomjcaQm01.

[4] Smith RW, Nelson DR. Fractures and other injuries from falls after an ice storm. Am J Emerg Med. 1998;16:52-55.

[5] Blindauer KM, Rubin C, Morse DL, McGeehin M. The 1996 New York blizzard: Impact on noninjury emergency visits. Am J Emerg Med. 1999;17:23-27.

[6] Broder J, Mehrotra A, Tintinalli J. Injuries from the 2002 North Carolina ice storm, and strategies for prevention. Injury. 2005;36:2126.

[7] Piercefield E, Wendling T, Archer P, Mallonee S. Winter stormrelated injuries in Oklahoma, January 2007. J Safety Res. 2011;42:27-32.

[8] Rajaram N, Hohenadel K, Gattoni L, Khan Y, Birk-Urovitz E, Li L, et al. Assessing health impacts of the December 2013 Ice storm in Ontario, Canada. BMC Public Health. 2016;16:544.

How to cite this article: Giovanni Landoni, Omar Saleh, Maurizio Migliori, Carlo Picco. Impact on the regional ambulance services and emergency departments of the 2017 freezing rain in Milan. Signa 
h Signa Vitae

Vitae. 2020;16(1):73-77. doi:10.22514/sv.2020.16.0010. 